\title{
La formación del profesional que atiende a los huéspedes mayores en establecimientos de asistencia social
}

\author{
The Training of the Professional Who Cares for Older People in Social \\ Assistance Establishments
}

\begin{abstract}
A formação do profissional que cuida de idosos em estabelecimentos de assistência social
\end{abstract}

Jaqueline Guadalupe Guerrero Ceh Universidad Autónoma de Campeche, México

jgguerre@uacam.mx https://orcid.org/0000-0002-2913-1309

Mariajose Sánchez de la Rosa Universidad Autónoma de Campeche, México al037362@uacam.mx https://orcid.org/0000-0002-4017-4051

\section{Resumen}

Ante el incremento de la cantidad de personas mayores, y el aumento del índice de soledad, surge la necesidad de que las personas acudan a un establecimiento de asistencia social para recibir atención profesional. Sin embargo, ¿cuál es la formación que tienen los cuidadores en las residencias geriátricas? La hipótesis aquí planteada fue que los cuidadores carecen de formación en la atención del paciente geriátrico. El objetivo del presente trabajo, por tanto, fue analizar el nivel de formación que tiene el personal en la atención que brinda al paciente geriátrico en las residencias de este tipo. Se trató de una investigación con un enfoque cualitativo, alcance descriptivo, transversal, y una muestra por conveniencia de 18 personas: integrada por 13 cuidadores de residencias geriátricas y cinco trabajadores del Instituto de Seguridad y Servicios Sociales de los Trabajadores del Estado (ISSSTE). Se desarrolló en 
tres etapas: diagnóstico situacional, diseño e impartición de un taller y propuesta de un diplomado para cuidadores. Respecto al perfil profesional de los participantes, $28 \%$ son enfermeros, $28 \%$ gerontólogos, $12 \%$ gerocultores, $17 \%$ técnicos y auxiliares de enfermería, $5 \%$ médicos, $5 \%$ licenciados en Economía y $5 \%$ cuenta con grado de bachillerato. Así, del total de la muestra, $29 \%$ carece de capacitación y $71 \%$ tiene alguna capacitación para su función. Como parte de la investigación, se aplicó un taller y se diseñó la propuesta de un diplomado para cuidadores. De entre las conclusiones, la hipótesis planteada se acepta, pues solo $40 \%$ tiene formación para la atención del paciente geriátrico; 71 \% tiene formación del área de la salud, y 29 \% carece de cursos en el área de atención del paciente geriátrico. Finalmente, el total de los participantes resultó satisfecho por el taller. No obstante, es importante continuar con la formación de cuidadores geriátricos en dos líneas: conocimiento y salud mental del cuidador.

Palabras clave: formación profesional, gerontología, hogares para ancianos, formación continua.

\section{Abstract}

Given the increase in the number of older people, and the increase in the loneliness index, the need arises for people to go to a social assistance establishment to receive professional care. However, what is the training that caregivers have in nursing homes? The hypothesis presented here was that caregivers lack training in geriatric patient care. The objective of this work, therefore, was to analyze the level of training that staff have in the care they provide to the geriatric patient in residences of this type. It was an investigation with a qualitative approach, descriptive, cross-sectional scope, and a convenience sample of 18 people. The sample was specifically integrated by 13 caregivers of nursing homes and five workers of the Instituto de Seguridad y Servicios Sociales de los Trabajadores del Estado (Issste). It was developed in three stages: situational diagnosis, the design and delivery of a workshop, and a diploma proposal for caregivers. Regarding the professional profile of the participants, $28 \%$ are nurses, $28 \%$ gerontologists, $12 \%$ geriatric nurses, $17 \%$ technicians and nursing assistants, $5 \%$ doctors, $5 \%$ graduates in Economics and $5 \%$ with a bachelor's degree. Thus, of the total sample, $29 \%$ lack training and $71 \%$ have some training for their function. As part of the research, a workshop was applied, and the proposal of a diploma for caregivers was 
made. Among the conclusions, the planned hypothesis is accepted, since only $40 \%$ have training in geriatric patient care; $71 \%$ have training in the health area, and $29 \%$ lack courses in the geriatric patient care area. Finally, the total of the participants was satisfied by the workshop. However, it is important to continue with the training of geriatric caregivers in two lines: knowledge and mental health of the caregiver.

Keywords: vocational training, gerontology, homes for the elderly, continuing education.

\section{Resumo}

Dado o aumento do número de idosos e o aumento do índice de solidão, surge a necessidade de as pessoas irem a um estabelecimento de assistência social para receber atendimento profissional. No entanto, qual é o treinamento que os cuidadores têm em casas de repouso? A hipótese apresentada aqui foi de que os cuidadores carecem de treinamento em assistência geriátrica ao paciente. O objetivo deste trabalho, portanto, foi analisar o nível de treinamento que a equipe possui no atendimento prestado ao paciente geriátrico em residências desse tipo. Trata-se de uma investigação de abordagem qualitativa, de caráter descritivo, transversal e com uma amostra de conveniência de 18 pessoas: composta por 13 cuidadores de asilos e cinco trabalhadores do Instituto de Segurança e Serviços Sociais para Trabalhadores do Estado (Issste). Foi desenvolvido em três etapas: diagnóstico situacional, desenho e entrega de uma oficina e proposta de um diploma para cuidadores. Quanto ao perfil profissional dos participantes, $28 \%$ são enfermeiros, $28 \%$ gerontologistas, 12\% geoculturadores, $17 \%$ técnicos e auxiliares de enfermagem, 5\% médicos, 5\% graduados em Economia e 5\% possuem diploma de bacharel. Assim, da amostra total, 29\% não possuem treinamento e $71 \%$ possuem algum treinamento para sua função. Como parte da pesquisa, um workshop foi aplicado e a proposta de um diploma para cuidadores foi elaborada. Entre as conclusões, a hipótese proposta é aceita, uma vez que apenas $40 \%$ possuem treinamento em assistência geriátrica ao paciente; $71 \%$ possuem treinamento na área da saúde e $29 \%$ não possuem cursos na área de atendimento ao paciente geriátrico. Finalmente, o total de participantes foi satisfeito pelo workshop. No entanto, é importante continuar com o treinamento de cuidadores geriátricos em duas linhas: conhecimento e saúde mental do cuidador.

Palavras-chave: formação profissional, gerontologia, lar de idosos, formação contínua. 


\section{Introducción}

Actualmente, todos los países están viviendo un incremento en la cantidad de viejos y ancianos. Esto debido a dos aspectos en común: disminución de la natalidad y el aumento de la esperanza de vida. Sin duda han quedado atrás los tiempos en que las familias eran numerosas, con 5, 10 o 15 hijos; ahora se encuentra muy arraigada la creencia de que "la familia pequeña vive mejor", y se tienen familias sin hijos, uno o dos hijos. Lo anterior trae una repercusión social importante que incide en el aumento del índice de soledad de las personas mayores, quienes, al estar solas, carecen de alguien responsable de su atención, motivo por el cual es necesario que en México existan espacios dignos y con personal del área de salud debidamente acreditado para la atención tanto de individuos con envejecimiento patológico como de aquellos sin patologías que deseen pasar la última etapa de su vida en un ambiente seguro y tranquilo.

Este estudio surge con el objetivo de analizar el nivel de formación que tiene el personal en la atención que brinda al paciente geriátrico en las residencias. Para el cumplimiento del objetivo del estudio, este artículo se encuentra integrado en cuatro partes: 1) introducción, donde se presenta la descripción de la situación problema y la selección de la literatura, base de análisis del sustento teórico, integrado por las etapas del desarrollo humano, la importancia de la formación del cuidador y los establecimientos de asistencia social; 2) método, aquí se explican los aspectos metodológicos aplicados en este estudio; 3) resultados, sección que inicia con el diseño y aplicación de un taller para cuidadores y termina con el diseño y propuesta de un diplomado para cuidadores, y 4) conclusión, con el análisis de la información obtenida, presentación de los resultados y evaluación de las repercusiones para el estado de la investigación en el tema. 


\section{Etapas del desarrollo humano}

Si bien existen múltiples clasificaciones de las etapas del ser humano, en este artículo se considera la propuesta por Erikson (citado en Morris y Maisto, 2011), quien ha concebido ocho: infancia (al año de edad), niñez temprana (1-3 años), niñez (3-6 años), niñez tardía (612 años), adolescencia (12-19 años), juventud (19-40 años), adultez (40-65 años) y madurez $(65+$ años). Para fines de este artículo interesa la última etapa de la edad, esto es, la madurez, la cual pertenece a la etapa psicosocial de integridad frente a desesperación y es caracterizada de la siguiente forma:

Esta etapa es una oportunidad para alcanzar la plenitud del yo, la aceptación de la vida propia, la sensación de que esta es completa y satisfactoria. La gente que ha obtenido una plena madurez al resolver los conflictos anteriores posee la integridad para enfrentar la muerte con un mínimo de temores. Para otros, este es un periodo de desesperación por la pérdida de los roles anteriores y de arrepentimiento por las oportunidades perdidas (Morris y Maisto, 2011, p. 407).

Dada las características de vida que se tiene en este siglo XXI, cada vez es mayor la población que en la etapa de la madurez viven un periodo de desesperación, vinculado a las complicaciones económicas y de salud que tienen.

En México, se realizó la encuesta intercensal en el año 2015 en cuyos resultados definitivos se hace referencia a lo siguiente:

La pirámide poblacional sigue la tendencia de reducir su base, mientras que continúa el crecimiento tanto en el centro como en la parte alta; lo que significa que la proporción de niñas, niños y adolescentes ha disminuido y se ha incrementado la de adultos y adultos mayores; estos últimos pasaron de $6.2 \%$ en 2010 a $7.2 \%$ en 2015. La edad mediana en México es de 27 años. (INEGI, 2015, pág. 1 -2)

En solo cinco años subió a nivel nacional el 1\%, ante lo cual se espera que para el 2050 se tripliquen las cifras de personas mayores, escenario que será evidente en todo el mundo.

El estado de Campeche, se ubica en el sureste de México, de acuerdo a la encuesta intercensal realizada por el INEGI en el 2015, tiene una población total de 899931 habitantes, de la población de personas adultas mayores ha ido al alza, pasando de $2.1 \%$ al $8.3 \%$, de 
acuerdo a datos del censo de población y vivienda del año 2010 del Instituto Nacional de Estadística y Geografía (INEGI). Las proyecciones que tiene el Consejo Nacional de Población (CONAPO, 2016) para este año 2020 es de 102776 personas adultas mayores, con una tasa de crecimiento anual cercano a $5 \%$. De los 11 municipios que integran el estado de Campeche, tres son los que tienen el mayor porcentaje de personas adultas mayores en su población: Palizada (9.05 \%), Calkiní (8.08 \%) y Tenabo (7.63 \%).

Los municipios que registran los mayores porcentajes de personas de 65 años y más en relación a su población total son: Palizada con el 9.05 por ciento, es decir, que 9 de cada cien paliceños tienen entre 65 años y más; seguido por el municipio de Calkiní con el 8.08 por ciento y Tenabo con el 7.63 por ciento, por mencionar los de mayor concentración con base a su población total. (CONAPO, 2016, pág. 20)

Sin embargo, como se ha observado, a pesar de ser los que tienen mayor población de personas envejecidas, ninguno de estos tres municipios tiene asilos para ancianos, por lo que su cuidado está a cargo de la familia.

\section{Importancia de la formación del cuidador}

En la Segunda Asamblea Mundial sobre el Envejecimiento, realizada en Madrid, España, del 8 al 12 de abril de 2002, como parte de los trabajos realizados en esta reunión, se emitió la Declaración Política y Plan de Acción Internacional de Madrid sobre el Envejecimiento. Allí, en su orientación prioritaria II, denominada El fomento de la salud y el bienestar en la vejez, se expresa lo siguiente:

Existe en todo el mundo una necesidad imperiosa de ampliar las oportunidades educacionales en geriatría y gerontología para todos los profesionales de la salud que atienden a personas de edad y de ampliar los programas educacionales sobre la salud y las personas de edad dirigidos a los profesionales del sector de servicios sociales (Organización de las Naciones Unidas [ONU], 2003, p. 38)

Debido a que es primordial conservar la vida de una persona, es importante que el personal de salud que atiende a los mayores se encuentre bien capacitado, especializado y actualizado. 


\section{Revista Iberoamericana de las Ciencias de la Salud}

Además de la importancia de la formación continua de los profesionales del área de la salud, que redunde en una atención con calidad y calidez hacia los viejos y ancianos, en este mismo documento, el Plan de Acción Internacional de Madrid sobre el Envejecimiento, se encuentra un aspecto también importante relacionado con el tema de la salud mental:

Las estrategias para hacer frente a esas enfermedades incluyen la medicación, el apoyo psicosocial, los programas de formación con un enfoque cognitivo, la formación de los familiares y profesionales que cuidan a los enfermos y las estructuras especiales de atención en régimen interno. (ONU, 2003, p. 39)

Así pues, desde el año 2002, ya se precisaba la preocupación por la salud mental y se recomendaba que debía iniciarse la formación de familiares y profesionales del área de la salud en el tema del deterioro cognitivo y las demencias, sobre todo en el aspecto de los tratamientos no farmacológicos. Entre las medidas se proponen diez incisos; aquí se destacan dos:

h) Promover la divulgación de información sobre los síntomas, el tratamiento, las consecuencias y el pronóstico de las enfermedades mentales; j) Impartir formación permanente sobre la detección y evaluación de todos los trastornos mentales y la depresión a los profesionales de la salud. (ONU, 2003, p. 40)

Como se aprecia, se enfatiza en la necesidad de trabajar en la formación de profesionales de la salud con conocimiento sobre la etiología de las demencias, con sus respectivos tratamientos farmacológicos y no farmacológicos, con procedimiento basados en evidencia que permita tanto atender al paciente como gerontologizar al familiar.

El equipo del área de la salud dedicado a la atención a las personas en las etapas de vejez y ancianidad debe ser integral; conformado por un médico especializado en geriatría, gerontólogo clínico, personal de enfermería, fisioterapeuta y terapeuta ocupacional, todos deben trabajar en conjunto para proponer tratamientos que permitan resolver las situaciones que limitan la independencia de la persona adulta mayor. Aún más, Millán (2006, p. 11) explica que en gerontología un equipo multidisciplinar ha de estar formado al menos por los siguientes profesionales: médico especializado en geriatría, psicólogo, enfermero, terapeuta ocupacional, fisioterapeuta, logopeda y trabajador social. Es de llamar la atención que no considera al gerontólogo como profesional que debe ser parte de este equipo, al menos en un primer momento así se percibe, por esa razón es importante que los gerontólogos continúen 
capacitándose para poder mejorar su desempeño profesional y, paulatinamente, se vayan insertando en ámbito laboral, en el que, gracias a sus conocimientos y actuación, se valore su importancia en la pronta recuperación del paciente geriátrico y adquiera visibilidad entre la gama de profesionistas del área en cuestión.

La capacitación continua del personal de salud incide en la formación del talento humano, lo cual permitirá que sean sensibles y ofrezcan un trato digno a la persona mayor. El equipo multidisciplinar debe trabajar de forma integral para lograr mayor eficiencia.

El Instituto Nacional de las Personas Adultas Mayores (INAPAM), en el año 2012, emitió un documento donde establece los modelos de atención y las habilidades del personal que atiende a las personas adultas mayores. Como profesional involucrado en el trabajo con las personas adultas mayores, debe reunir las siguientes características:

- Comportamiento profesional

- $\quad$ Conocimiento del tema

- $\quad$ Calidez y gentileza

- Empatía

- Respeto

- $\quad$ Autenticidad, Flexibilidad

- $\quad$ Sentido del humor

- $\quad$ Lenguaje adecuado

- $\quad$ Tono de voz apropiado

- Habilidades pedagógicas

- $\quad$ Habilidad de escucha

- $\quad$ Habilidad de síntesis

- $\quad$ Manejo de situaciones familiares (INAPAM, 2012, p. 50)

Igualmente, el INAPAM (2012) desglosa las funciones principales que deben tener estos profesionales:

- $\quad$ Realizar la planeación de objetivos y su evaluación

- Lograr un cambio de actitud en la persona adulta mayor, incidiendo en su entorno social y familiar

- $\quad$ Respetar la personalidad de cada uno de los miembros y reconocer su contribución al desarrollo del trabajo 
- Fomentar el espíritu grupal por medio de sentimientos de amistad

- Brindar atención individualizada y grupal

- $\quad$ Promover y fortalecer las relaciones interpersonales por medio de la orientación y educación

- Mantener el contacto continuo con los adultos mayores y con el personal interdisciplinario para el buen desarrollo de las actividades

- Impulsar un trabajo independiente y autogestivo del grupo (pp. 51-51).

Por su parte, en el Diario Oficial de la Federación [DOF] (12 de septiembre de 2013) se publicó el "PROY-NOM-031-SSA3-2009 Asistencia social. Prestación de servicios de asistencia social a adultos y adultos mayores en situación de riesgo y vulnerabilidad", donde, en el punto seis, dedicado a los recursos humanos, se expresa lo siguiente:

6.4. Las instituciones de asistencia social de los sectores público, social y privado, por medios propios o a través de terceros, serán responsables de capacitar al personal en temas relacionados con la vejez y educación continua en la materia, preferentemente una vez al año. (párr. 24)

De esta forma, se confirma el deber que tienen las instituciones de gerontologizar a su personal y a los familiares de las personas que atienden. De hecho, en dicho proyecto de norma queda establecido que mínimo una vez al año se deben realizar estas actividades de formación continua.

El compromiso está normado, sin embargo, pocos son los establecimientos de asistencia social que cumplen, si acaso el responsable es quien se encuentra capacitado. Como ya se mencionó, la intención del PROY-NOM-031-SSA3-2009 es ofrecer al personal al menos una actualización al año para garantizar que la atención de las personas sea con calidad y calidez

\section{Los establecimientos de asistencia social}

Ante el incremento de la cantidad de personas mayores, aunado al aumento del índice de soledad, surge la necesidad de que las personas acudan a un establecimiento de asistencia social, ya sea de forma permanente o temporal, para recibir atención en esta etapa de su vida. Los países desarrollados, como Estados Unidos, han implementado una oferta de seguros, entre los que se encuentran Medicare o Medicaid, que las personas toman en consideración, 
acorde a sus posibilidades, analizando los costos, ya que, dependiendo del precio pactado, es la cobertura que se adquiere. En ese país, Estados Unidos, los espacios de cuidado tienen el concepto de ofrecer sus servicios en dos líneas de atención: una de ellas enfocada a las personas convalecientes, quienes, después de una intervención quirúrgica, requieren de cuidados especiales para su pronta rehabilitación; la otra línea está dirigida a personas que necesitan cuidados debido a su envejecimiento patológico o cronológico, que pertenecen a una familia que no puede dedicarle atención debido a sus compromisos laborales; también existe el caso de que carezca de familiares que puedan hacerse cargo de su persona, para darle la atención que merece debido a la fragilidad que presenta el anciano.

En México se carece de esta oferta de seguros. Tal vez los empresarios no se animan a invertir en este sector debido a la limitada percepción económica que tienen los mexicanos y latinoamericanos en su conjunto. El Inapam, en la Ciudad de México (antes Distrito Federal) ha implementado desde principios de este siglo XXI modelos de atención enfocados en las personas mayores, considerados en conjunto un sector de protección. De igual manera, han surgido jóvenes emprendedores que, buscando una oportunidad de trabajo, se han convertido en pequeños empresarios y están haciendo el esfuerzo para abrir espacios dedicados a la atención profesional de las personas adultas mayores; espacios también conocidos como: estancias geriátricas, residencias geriátricas, asilos, hogar de ancianos, establecimientos de asistencia social, entre otros nombres.

En el estado de Campeche, México, un grupo de jóvenes emprendedores, integrado por egresados de la licenciatura en Gerontología, ha incursionado en el mercado del cuidado de las personas mayores, y ha adaptado casas para ofrecer sus servicios al público, cuidando los requisitos sanitarios básicos, con poco personal profesional y mínima capacitación en el área de la gerontogeriatría. Es importante aclarar que este escenario se debe a que se carece de apoyo gubernamental y se subsiste solo con los recursos proveniente de las cuotas mensuales de los huéspedes. Algunos integrantes del área de enfermería son otros que también han incursionado como responsables de espacios para la atención de personas mayores.

De acuerdo con los datos de MedlinePlus (5 de diciembre de 2018), los espacios que ofertan servicios de atención permanente y temporal deben considerar entre sus compromisos: ofrecer permanentemente una "atención médica y de enfermería de rutina, ser asistidos en su aseo personal, recibir terapia física y ocupacional, así como todos sus 
alimentos" (párr. 2). También se recomienda que antes de tomar una decisión para ingresar al familiar se deben examinar las opciones, tomando en cuenta la cercanía del lugar, el trato digno y la atención que ofrecen, informarse de los servicios que incluye el costo, los permisos sanitarios y de las autoridades de protección civil para su funcionamiento, indagar sobre la formación que tienen las personas que estarán a cargo de su familiar.

Como lo adelantábamos líneas arriba, el INAPAM ofrece en la Ciudad de México cinco modelos de atención, a saber: albergues, residencias de día, centros de atención integral, centros culturales y clubes, todos ellos coinciden en que el primer requisito para la atención es la edad que debe tener la persona: 60 años y más de edad. Seguidamente se describen los objetivos de cada uno de los modelos:

- Los albergues tienen la intención de proteger y atender a las personas que lo requieran por su condición de vulnerabilidad. Para su ingreso deben tener estado de abandono, ser funcionales, carecer de enfermedades psiquiátricas; se les ofrece una estancia permanente y una atención digna (INAPAM, 2012, p. 61).

- Las residencias de día atienden a personas que no pueden permanecer solas en sus hogares, por lo que, mientras sus familiares están trabajando, ellos están en un espacio en el que socializan y comparten sus experiencias, a través de las actividades de terapia ocupacional (INAPAM, 2012, p. 69). Los asistentes son atendidos por un equipo interdisciplinar, que también se ocupa de la promoción de su salud física y mental.

- $\quad$ Los centros de atención integral son "unidades donde se proporciona atención médica integral especializada con el objetivo de conservar o recuperar la salud mediante acciones preventivas, curativas y de rehabilitación" (INAPAM, 2012, p. 77). Se trata de espacios que ofrecen servicios de calidad a bajo costo, especializados en la atención de la persona mayor. Los servicios que se ofrecen son: consulta externa, atención psicológica, odontología, laboratorio dental, servicios de laboratorio clínico y de gabinete, cardiología, optometría, rehabilitación, orientación nutricional, otorrinolaringología, audiología, audiometría, dermatología, endocrinología, geriatría, ginecología, ortopedia, reumatología, trabajo social, afiliación al Inapam, bolsa de trabajo y asesoría jurídica.

- Los centros culturales "son espacios que ofrecen formación y desarrollo humano a través de educación, capacitación, manualidades, actividades culturales, recreativas y deportivas" (INAPAM, 2012, p. 87). Tienen la finalidad de mantener la 
funcionalidad y autonomía, mejorar la capacidad física, socializar. Además, en estos espacios también se les ofrece consulta médica y atención psicológica.

- Los clubes "son espacios comunitarios donde se promueven actividades que ayudan a las personas adultas mayores a su socialización, lo que contribuye a mejorar su autoestima y a continuar integrados a su comunidad" (INAPAM, 2012, p. 93). Entre los servicios que se ofrecen están: atención médica, orientación jurídica, recreación, voluntariado, psicología, educación continua, bolsa de trabajo y preparación académica.

En el estado de Campeche, México, se carece de todos estos modelos diseñados y promovidos por el Inapam; solo se cuenta con dos asilos formales a cargo de Desarrollo Integral de la Familia (DIF), uno ubicado en Candelaria y el otro en la Ciudad de San Francisco de Campeche; en la ciudad de Escárcega existe una casa habilitada como asilo que recibe apoyo del H. Ayuntamiento. Esto se debe probablemente a la falta de presupuesto asignado al Inapam, el cual trabaja con apenas tres personas en la delegación estatal y hasta el momento se carece de la figura de delegado estatal, quien tiene el compromiso de atender y cumplir con sus responsabilidades fundamentadas en la Ley de los Derechos de las Personas Adultos Mayores (LDPAM), emitida por el Gobierno Federal (2008). En cuanto al INAPAM, en este documento se señala lo siguiente:

Este organismo público es rector de la política nacional a favor de las personas adultas mayores, teniendo por objeto general coordinar, promover, apoyar, fomentar, vigilar y evaluar las acciones públicas, estrategias y programas que se deriven de ella, de conformidad con los principios, objetivos y disposiciones contenidas en la presente Ley (Gobierno Federal, 2008, p. 14).

Es evidente que en la ley se encuentra plasmada la importancia del Inapam, sin embargo, en la realidad no se percibe al carecer de presupuesto propio, motivo por el cual se apoya en los estudiantes que acuden a esta institución para solicitar espacio para realizar sus prácticas clínicas y servicio social, provenientes de instituciones educativas como la Escuela Técnica de Gericultura y la licenciatura en Gerontología. Esta situación se ha visto más marcada desde el sexenio anterior (2012-2018); confiamos en que este nuevo Gobierno aporte mejor presupuesto a la atención de las personas de edad, pero no a través del asistencialismo. 


\section{Revista Iberoamericana de las Ciencias de la Salud}

En México existen normas oficiales que regulan los modelos de asistencia social y permanente. A continuación, se describen las tres más relevantes relacionadas con el tema de este estudio:

La "NOM-031-SSA3-2009, Asistencia social. Prestación de servicios de asistencia social a adultos y adultos mayores en situación de riesgo y vulnerabilidad, que deben cumplir quienes presten servicios de asistencia social", cuyo objetivo es el puesto a continuación:

Esta norma tiene por objeto establecer las características de funcionamiento, organización e infraestructura que deben observar los establecimientos de los sectores público, social y privado, que presten servicios de asistencia social a adultos y adultos mayores en situación de riesgo y vulnerabilidad (DOF, 13 de septiembre de 2012, párr. 8)

- La "NOM-004-SSA3-2012, Del expediente clínico" tiene como objetivo establecer "los criterios científicos, éticos, tecnológicos y administrativos obligatorios en la elaboración, integración, uso, manejo, archivo, conservación, propiedad, titularidad y confidencialidad del expediente clínico" (DOF, 5 de octubre de 2010, párr. 17). Es sumamente importante que todo el personal del área de la salud conozca, aplique e interprete perfectamente lo aquí presentado en las normas.

- $\quad$ Finalmente, la "NOM-030-SSA3-2013, Que establece las características arquitectónicas para facilitar el acceso, tránsito, uso y permanencia de las personas con discapacidad en establecimientos para la atención médica ambulatoria y hospitalaria del Sistema Nacional de Salud", la cual tiene el fin de "establecer las características arquitectónicas mínimas, que deben cumplir los establecimientos para la atención médica ambulatoria y hospitalaria del Sistema Nacional de Salud, para facilitar el acceso, tránsito, uso y permanencia de las personas con discapacidad" (DOF, 12 de septiembre de 2013, párr. $11)$.

Todas estas normas oficiales deben ser del conocimiento del personal de salud que trabaja en los establecimientos de asistencia social, permanente y temporal. También es importante que las autoridades de protección civil, del Inapam y de la Secretaría de Salud realicen al menos dos veces al año la supervisión correspondiente, para garantizar la correcta aplicación de protocolos de actuación, y evitar así posibles contingencias que pongan en riesgo la vida e integridad física de las personas. 
Esta investigación integró a los espacios públicos y privados que ofrecen este tipo de atención en el estado de Campeche. La pregunta de investigación fue la siguiente: ¿cuál es la formación que tienen los cuidadores de los huéspedes de residencias geriátricas? Y la hipótesis: los cuidadores de las residencias geriátricas tienen el perfil del área de la salud, pero carecen de formación en la atención del paciente geriátrico.

\section{Materiales y método}

Este estudio se realizó basado en un enfoque cualitativo, con alcance descriptivo. Como parte de los aspectos metodológicos, primero se analizó el número de establecimientos de asistencia social reconocidos por la delegación Campeche del Inapam. Con estos datos se procedió a integrar el protocolo de investigación que se presentó a la directora académica de la Facultad de Enfermería para solicitar los oficios de invitación a todos los establecimientos de asistencia social del estado de Campeche. Una vez aprobado el protocolo, se realizó la entrega de los oficios de invitación, y se continuó con el proceso para el desarrollo del taller (figura 1). 
Figura 1. Proceso de impartición del taller

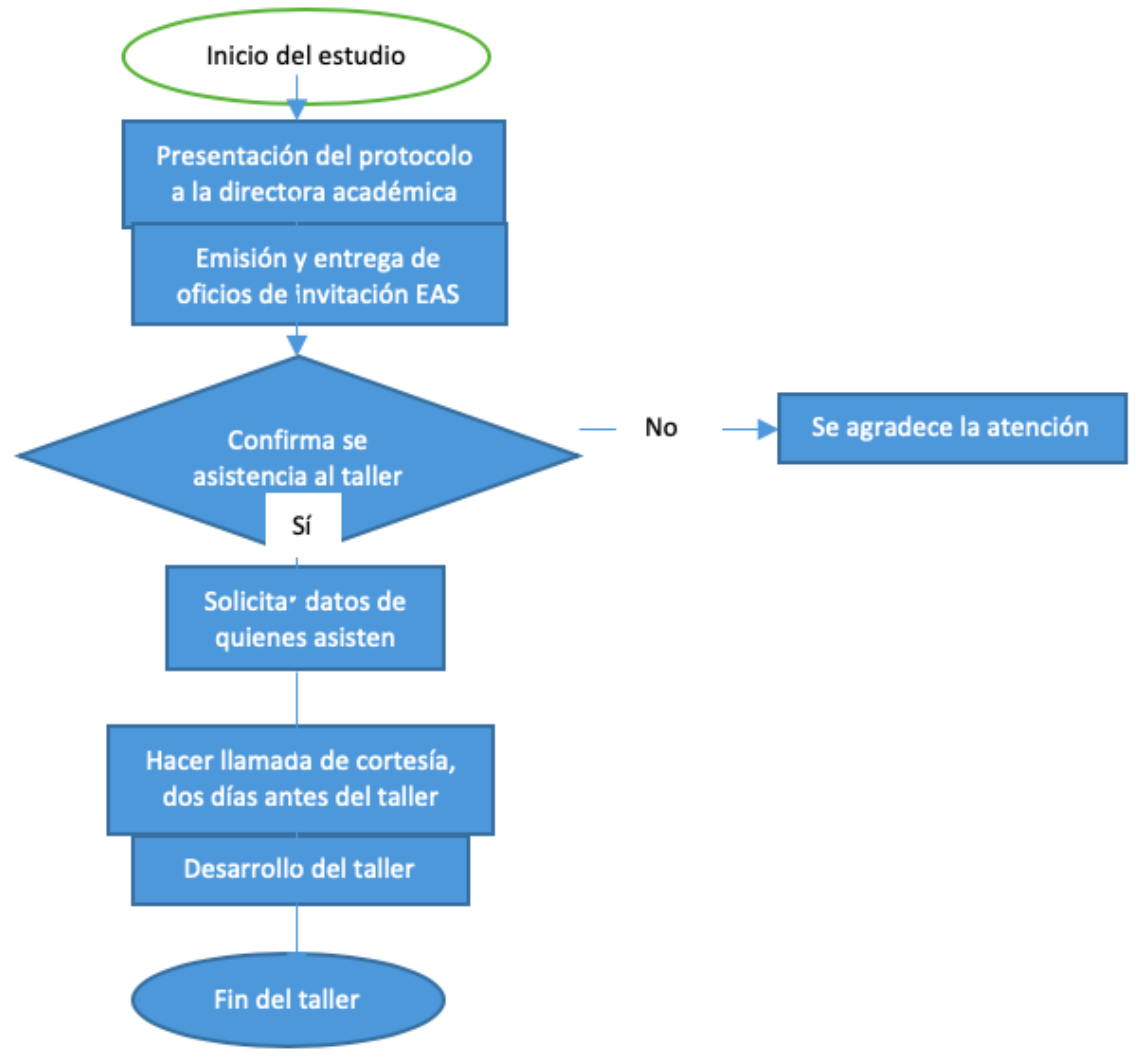

Fuente: Elaboración propia, 2019.

Al inicio del desarrollo del taller, se entregó a cada uno de los participantes un cuestionario de elaboración propia, compuesto de preguntas abiertas, el cual se diseñó con la intención de conocer información básica de los participantes, tales como: lugar de trabajo, formación profesional, así como los cursos del área geronto-geriátrica que ha cursado, precisando cuántos al año. La duración del estudio fue de 10 meses: inició en enero y concluyó en octubre de 2018.

\section{Procedimiento para la selección de la muestra}

La muestra fue por conveniencia, y estuvo integrada por 18 personas, de las cuales 13 se desempeñan como cuidadores en residencias geriátricas y cinco son trabajadores del Instituto de Seguridad y Servicios Sociales de los Trabajadores del Estado (ISSSTE). La investigación se desarrolló en tres etapas: diagnóstico situacional, programa de formación (impartición de taller) y propuesta de diplomado para cuidadores. Se realizó en los meses de enero a julio de 2018 . 


\section{Revista Iberoamericana de las Ciencias de la Salud}

En el mes de febrero de 2018, se emitieron las invitaciones a las residencias geriátricas y asilos, ubicados en el estado de Campeche, México, cuyos trabajadores tuvieron limitación para asistir por cuestiones económicas. Debido a ello, se invitó al personal de la delegación estatal del ISSSTE para que participaran en el taller. En total participaron 18 personas, como ya se mencionó, de las cuales 13 se desempeñan como cuidadores en cinco residencias geriátricas y cinco trabajan con personas mayores en el ISSSTE. En las figuras 2 y 3 se detalla el programa del taller aplicado: "Mejorando la atención del paciente geriátrico", desarrollado los días 14 y 15 de junio de 2018. A los participantes se les entregó constancia con valor curricular y avalado por la Universidad Autónoma de Campeche.

Figura 2. Programa de taller, primer día

\begin{tabular}{|c|c|c|c|c|c|c|}
\hline \multicolumn{7}{|c|}{ UNIVERSIDAD AUTÓNOMA DE CAMPECHE } \\
\hline & \multicolumn{6}{|c|}{ FACULTAD DE ENFERMERIAA } \\
\hline \multicolumn{7}{|c|}{ Taller: "Mejorando la atención del paciente geriátrico" } \\
\hline \multicolumn{7}{|c|}{ PROYECTO DE INVESTIGACIÓN: Importancia de la formación gerontogeriátrica del personal que atiende al paciente en las residencias. } \\
\hline \multicolumn{7}{|c|}{ RESPONSABLE INSTITUCIONAL: Dra. Jaqueline Guadalupe Guerrero Ceh } \\
\hline \multicolumn{7}{|c|}{ DÍA UNO: JUEVES 14 DE JUNIO DE 2018} \\
\hline HORA & ACTIVIDAD & OB.JETIVO & MATERIAL & RESPONSABLE & ESPACIO & OBSERVACIONES \\
\hline $08: 00$ & Registro & Identificar a los asistentes & Listas de asistencia & $\begin{array}{l}\text { Dra. Jaqueline } \\
\text { Guerrero }\end{array}$ & Aula & \\
\hline 08:10 & Bienvenida & $\begin{array}{l}\text { Agradecer la presencia a la } \\
\text { actividad. }\end{array}$ & Ninguno & $\begin{array}{l}\text { Dra. Jaqueline } \\
\text { Guadalupe } \\
\text { Guerrero Ceh }\end{array}$ & Aula & $\begin{array}{l}\text { Se da la bienvenida y se } \\
\text { explica la dinámica de } \\
\text { trabajo, así como la } \\
\text { intención del taller. }\end{array}$ \\
\hline 08:15 & $\begin{array}{l}\text { Conociendo nuestras } \\
\text { instituciones que atienden a } \\
\text { la persona mayor. }\end{array}$ & 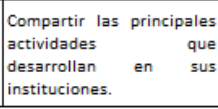 & $\begin{array}{l}\text { Computadora, } \\
\text { cañón, } \\
\text { instrumentos de } \\
\text { dx, papel bond, } \\
\text { marcadores. }\end{array}$ & $\begin{array}{l}\text { Dra. Jaqueline } \\
\text { Guadalupe } \\
\text { Guerrero Ceh y } \\
\text { Mtra. Ana Rosa } \\
\text { CanValle }\end{array}$ & Aula & $\begin{array}{|lr|}\text { Se distribuye r los } \\
\text { instrumentos } & \text { de } \\
\text { diagnóstico, el trabajo } \\
\text { es por institución. }\end{array}$ \\
\hline $09: 40$ & \multicolumn{4}{|c|}{ Receso } & & Se ofrece cofee breake \\
\hline 10:00 & $\begin{array}{l}\text { Sindrome del desgaste del } \\
\text { cuidador }\end{array}$ & $\begin{array}{l}\text { Aplicar las acciones para } \\
\text { controlar el síndrome del } \\
\text { cuidador y mantener el } \\
\text { buen trato al paciente. }\end{array}$ & Aula & $\begin{array}{l}\text { Mtra. Ana Rosa } \\
\text { Can Valle }\end{array} \mid$ & Aula & \\
\hline $12: 00$ & $\begin{array}{l}\text { La formación continua del } \\
\text { talento humano }\end{array}$ & $\begin{array}{l}\text { Identificar las plataformas } \\
\text { que ofrecen capacitación } \\
\text { en el ámbito geronto- } \\
\text { geriátrice. }\end{array}$ & \begin{tabular}{|l|} 
Sala de computo \\
con acceso a \\
Internet
\end{tabular} & \begin{tabular}{|l|} 
Dra. Jaqueline \\
Guadalupe \\
Guerrero Ceh
\end{tabular} & \begin{tabular}{|l|} 
Sala de cómputo \\
con acceso a \\
Internet
\end{tabular} & \\
\hline 13:00 & \multicolumn{4}{|c|}{ Receso } & & Se ofrece cofee breake \\
\hline 15:00 & $\begin{array}{l}\text { Acciones preventivas para el } \\
\text { manejo de residentes }\end{array}$ & 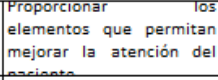 & Aula & $\begin{array}{|lr|}\text { Mtra. } & \text { Manuela } \\
\text { del } & \text { Jesús } \\
\text { Vallejos Tun }\end{array}$ & Aula & \\
\hline $17: 00$ & $\begin{array}{l}\text { Movilizaciones para prevenir } \\
\text { UPP }\end{array}$ & $\begin{array}{l}\text { Saber aplicar las acciones } \\
\text { preventivas de las UPP }\end{array}$ & 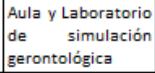 & $\begin{array}{l}\text { Dr. José Misael } \\
\text { Uc Ucán }\end{array}$ & 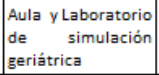 & \\
\hline $19: 00$ & \multicolumn{6}{|c|}{ FIN DE LASESIÓN } \\
\hline
\end{tabular}

Fuente: Elaboración propia 
Figura 3. Programa de taller, segundo día

\begin{tabular}{|c|c|c|c|c|c|c|}
\hline \multicolumn{7}{|c|}{ UNIVERSIDAD AUTÓNOMA DE CAMPECHE } \\
\hline \multirow{2}{*}{\multicolumn{7}{|c|}{ Taller: "Mejorando la atención del paciente geriátrico" }} \\
\hline & & \multicolumn{4}{|c|}{ Taller: "Mejorando la atención del paciente geriátrico" } & taie: \\
\hline \multicolumn{7}{|c|}{ PROYECTO DE INVESTIGACIÓN: Importancia de la formación gerontogeriátrica del personal que atiende al paciente en las residencias. } \\
\hline \multicolumn{7}{|c|}{ RESPONSABELE INSTITUCIONAGL: Dra. Jaqueline Guadalupe Guerrero Ceh } \\
\hline & & & & & & \\
\hline \multicolumn{7}{|c|}{ DIA DOS: VIERNES 15 DE JUNIO DE 2018} \\
\hline HORA & ACTIVIDAD & QB.JETIVO & MATERIAL & RESPONSABLE & ESPACIO & OBSERVACIONES \\
\hline 08:00 & Registro & Identificar a los asistentes & Listas de asistencia & $\begin{array}{l}\text { Dra. Jaqueline } \\
\text { Guerrero }\end{array}$ & Aula & \\
\hline 08:10 & $\begin{array}{l}\text { Sindrome del desgaste del } \\
\text { cuidador }\end{array}$ & $\begin{array}{l}\text { Aplicar las acciones para } \\
\text { controlar el sindrome del } \\
\text { cuidador y mantener el } \\
\text { buen trato al paciente. }\end{array}$ & Aula & $\begin{array}{l}\text { Mtra. Ana Rosa } \\
\text { Can Valle }\end{array}$ & Aula & $\begin{array}{l}\text { Se da la bienvenida y se } \\
\text { explica la dinámica de } \\
\text { trabajo, así como la } \\
\text { intención del taller. }\end{array}$ \\
\hline 10:00 & \multicolumn{4}{|c|}{ Receso } & & Se ofrece cofee breake \\
\hline 10:20 & $\begin{array}{l}\text { La formación continua del } \\
\text { talento humano }\end{array}$ & $\begin{array}{l}\text { Identificar las plataformas } \\
\text { que ofrecen capacitación } \\
\text { en el ámbito geronto- } \\
\text { geriátrico. }\end{array}$ & $\begin{array}{l}\text { Sala de cómputo } \\
\text { con acceso a } \\
\text { Internet }\end{array}$ & $\begin{array}{l}\text { Dra. Jaqueline } \\
\text { Guadalupe } \\
\text { Guerrero Ceh }\end{array}$ & $\left|\begin{array}{lrr}\text { Aula } & - \text { Sala } & \text { de } \\
\text { cómputo } & \text { con } \\
\text { acceso a Internet }\end{array}\right|$ & \\
\hline 13:00 & \multicolumn{4}{|c|}{ Receso } & & Se ofrece cofee breake \\
\hline 15:00 & $\begin{array}{l}\text { La formación continua del } \\
\text { talento humano }\end{array}$ & $\begin{array}{l}\text { Identificar las plataformas } \\
\text { que ofrecen capacitación } \\
\text { en el ámbito geronto- } \\
\text { geriátrico. }\end{array}$ & $\begin{array}{l}\text { Sala de cómputo } \\
\text { con acceso a } \\
\text { Internet }\end{array}$ & $\begin{array}{l}\text { Dra. Jaqueline } \\
\text { Guadalupe } \\
\text { Guerrero Ceh }\end{array}$ & $\mid \begin{array}{l}\text { Sala de cómputo } \\
\text { con acceso a } \\
\text { Internet-Aula }\end{array}$ & \\
\hline 16:00 & $\begin{array}{l}\text { Movilizaciones para prevenir } \\
\text { UPP }\end{array}$ & $\begin{array}{l}\text { Saber aplicar las acciones } \\
\text { preventivas de las UPP }\end{array}$ & 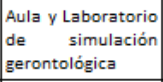 & $\begin{array}{l}\text { Dr. José Misael } \\
\text { Uc Ucán }\end{array}$ & 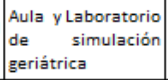 & \\
\hline 18:00 & $\begin{array}{l}\text { Acciones preventivas para el } \\
\text { manejo de residentes }\end{array}$ & $\begin{array}{l}\text { Propocionar los elementos } \\
\text { que permitan mejorar la } \\
\text { atención del paciente }\end{array}$ & Aula & $\begin{array}{lr}\text { Mtra. } & \text { Manuela } \\
\text { del } & \text { Jesús } \\
\text { Vallejos Tun }\end{array}$ & Aula & \\
\hline 20:00 & \multicolumn{6}{|c|}{ CLAUSURA Y ENTREGA DE CONSTANCIAS } \\
\hline
\end{tabular}

Fuente: Elaboración propia, 2019

\section{Resultados}

Al realizar la investigación de campo, para la ubicación de los establecimientos de asistencia social que ofrecen atención permanente y temporal en el estado de Campeche, se encontraron 13 espacios de atención permanente para las personas en la etapa de madurez, de los cuales uno está en Escárcega, dos en Ciudad del Carmen, uno en Candelaria, uno en Hopelchén y ocho en Campeche. En la tabla 1 se presentan los municipios que tienen espacios para la atención temporal y permanente de las personas ancianas, así como el tipo de servicio que ofrecen (privada o pública), incluyendo el porcentaje de población mayor de 60 años en cada uno. 
Tabla 1. Relación de estancias en el estado de Campeche

\begin{tabular}{|c|c|c|c|c|}
\hline Campeche & Carmen & Candelaria & Escárcega & Hopelchén \\
\hline $8.3 \%$ & $6.4 \%$ & $8.0 \%$ & $7.6 \%$ & $8.7 \%$ \\
\hline $\begin{array}{l}\text { Hogar de ancianos } \\
\text { Dolores Lanz de } \\
\text { Echeverría } \\
\text { (Público) }\end{array}$ & $\begin{array}{c}\text { Hogar de } \\
\text { ancianos Villa } \\
\text { Merced }\end{array}$ & $\begin{array}{ll}\begin{array}{l}\text { Hogar } \\
\text { ancianos }\end{array} & \text { de } \\
\text { María } & \text { del } \\
\text { Carmen } & \\
\text { Montero } & \text { de } \\
\text { Hurtado } & \\
\text { (Público) } & \end{array}$ & $\begin{array}{l}\text { Hogar de } \\
\text { ancianos } \\
\text { Alba María } \\
\text { y Sofía } \\
\text { (Público) }\end{array}$ & $\begin{array}{l}\text { Hogar de } \\
\text { ancianos } \\
\text { Abelardo } \\
\text { Lara Negrón } \\
\text { (Público) }\end{array}$ \\
\hline $\begin{array}{l}\text { Residencia y casa de } \\
\text { día para adultos } \\
\text { mayores Hogar del } \\
\text { Abuelo } \\
\text { (Privado) }\end{array}$ & $\begin{array}{l}\text { El Mundo de } \\
\text { los Abuelos }\end{array}$ & & & \\
\hline $\begin{array}{l}\text { Residencia y guardería } \\
\text { geriátrica Casa Grande } \\
\text { (Privado) }\end{array}$ & & & & \\
\hline $\begin{array}{c}\text { Residencia geriátrica } \\
\text { La Casa de las } \\
\text { Abuelas } \\
\text { (Privado) }\end{array}$ & & & & \\
\hline $\begin{array}{c}\text { Centro gerontológico } \\
\text { Fengari } \\
\text { (Privado) }\end{array}$ & & & & \\
\hline $\begin{array}{c}\text { Guardería y casa } \\
\text { hogar Narci }\end{array}$ & & & & \\
\hline $\begin{array}{l}\text { Estancia Los años } \\
\text { Dorados } \\
\text { (Privado) }\end{array}$ & & & & \\
\hline $\begin{array}{c}\text { Centro de día y } \\
\text { estancia permanente } \\
\text { Vital Care } \\
\text { (Privado) }\end{array}$ & & & & \\
\hline \multicolumn{5}{|c|}{ Estancia temporal } \\
\hline $\begin{array}{l}\text { Centro de atención } \\
\text { integral al adulto } \\
\text { mayor. Estancia: La } \\
\text { Alegría de Vivir } \\
\text { (Público) }\end{array}$ & $\begin{array}{l}\text { Centro } \\
\text { gerontológico: } \\
\text { El Mundo de } \\
\text { los Abuelos }\end{array}$ & & & \\
\hline $\begin{array}{l}\text { Centro de atención } \\
\text { integral del adulto } \\
\text { mayor (Caiam - } \\
\text { Issstecam) } \\
\text { (Público) }\end{array}$ & $\begin{array}{l}\text { Estancia para el } \\
\text { adulto mayor: } \\
\text { Héroes de } \\
\text { Nacozari }\end{array}$ & & & \\
\hline
\end{tabular}




\begin{tabular}{|c|l|l|l|l|}
\hline $\begin{array}{c}\text { Centro recreativo y } \\
\text { asistencia al adulto } \\
\text { mayor }\end{array}$ & & & & \\
\hline $\begin{array}{c}\text { Centro de } \\
\text { estimulación } \\
\text { cognitiva: Soy Mayor }\end{array}$ & & & & \\
\hline
\end{tabular}

Fuente: Elaboración propia, con datos proporcionados por la delegación del INAPAM en Campeche e INEGI (entrevista realizada al Delegado del INAPAM el 27 de septiembre de 2018)

Como parte del compromiso y responsabilidad social que tiene la Universidad Autónoma de Campeche, México, se procedió al diseño de un taller dirigido a los cuidadores de las residencias geriátricas que ofrecen sus servicios en el estado de Campeche, México, con el objetivo de contribuir a una mejor atención del paciente en las residencias geriátricas privadas. A través de la experiencia y análisis de los temas geronto-geriátricos básicos, se consideró en un inicio una participación máxima de 30 personas. La actividad de capacitación tuvo una duración total de 20 horas, dividida en cuatro segmentos de cinco horas cada uno (matutino y vespertino). Cada uno de los cuatro temas tiene una duración de cuatro horas, un total de dieciséis horas. Las otras cuatro horas, se trabajarán en actividades elaboración del diagnóstico situacional de las residencias geriátricas, la evaluación y la entrega de constancias. Los temas a desarrollar fueron: formación continua del talento humano, síndrome del desgaste del cuidador, acciones preventivas para el manejo de residentes y movilizaciones para prevenir úlceras por presión (UPP). 
Tabla 2. Estructura del taller impartido

\begin{tabular}{|c|}
\hline Talle \\
\hline Responsable institucional: Dra \\
\hline $\begin{array}{l}\text { Objetivo: Este taller se realiza con la intención de contribuir en una mejor atención del } \\
\text { paciente de las residencias geriátricas privadas, a través de la experiencia y análisis de los } \\
\text { temas geronto-geriátricos básicos. }\end{array}$ \\
\hline $\mathrm{Pa}$ \\
\hline $\begin{array}{l}\text { Estructura: La actividad tiene una duración total de veinte horas, dividida en cuatro } \\
\text { segmentos de cinco horas en cada turno matutino y vespertino. Cada uno de los cuatro } \\
\text { temas, tiene una duración de cuatro horas, un total de dieciséis horas. Las otras cuatro } \\
\text { horas, se trabajarán en actividades elaboración del diagnóstico situacional de las } \\
\text { residencias geriátricas, la evaluación y la entrega de constancias. Seguidamente se } \\
\text { describen los temas a desarrollar: }\end{array}$ \\
\hline $\begin{array}{l}\text { 1) La formación continua del talento humano. Objetivo: Identificar la oferta educativa } \\
\text { de las plataformas online en el ámbito geronto-geriátrico. }\end{array}$ \\
\hline $\begin{array}{l}\text { 2) Síndrome del desgaste del cuidador. Objetivo: Aplicar las acciones para controlar } \\
\text { el síndrome del cuidador, manteniendo una buena calidad de vida, para ofrecer un } \\
\text { buen trato al paciente. }\end{array}$ \\
\hline $\begin{array}{l}\text { 3) Acciones preventivas para el manejo de residentes. Objetivo: Proporcionar los } \\
\text { elementos que permitan mejorar la atención del paciente en una residencia } \\
\text { geriátrica. }\end{array}$ \\
\hline $\begin{array}{l}\text { 4) Movilizaciones para prevenir UPP. Objetivo: Conocer y aplicar las acciones } \\
\text { preventivas de las UPP en el paciente geriátrico. }\end{array}$ \\
\hline
\end{tabular}

Fuente: Elaboración propia, 2019.

En la primera parte del taller impartido, se realizó una actividad que permitió conocer las necesidades de capacitación que tienen. Con esta información se procedió a realizar una propuesta denominada Diplomado para cuidadores de personas envejecidas. Ha sido diseñada de acuerdo con las necesidades expresadas y observadas en las familias y los establecimientos de asistencia social en la ciudad de San Francisco de Campeche, Campeche, México, por lo que, en un primer momento, está enfocada en las personas que son cuidadoras informales. Entre los instructores están considerados: docentes de gerontología, un geriatra, una especialista en medicina familiar, gerontólogos, así como el equipo de auxiliares 
geriátricas del Consejo Mexicano de Geriatría y Gerontología de Puebla y del Centro de Rehabilitación y Educación Especial (CREE) Campeche experto en el taller de uso y manejo de sillas de ruedas; aún es necesario establecer los convenios necesarios con estas dos instancias expertas.

La propuesta del diplomado está integrada por nueve módulos, con valor curricular. Cada módulo tiene una duración de 20 horas, de las cuales 16 horas son presenciales y 4 horas autoadministrables, un total de 180 horas. Los módulos son:

1) Principales enfermedades en la persona mayor que originan sus cuidados.

2) Salud mental en los cuidadores.

3) El cuidado del cuidador.

4) Tanatología y su impacto en la persona: cuidador y familia.

5) Técnicas de cuidado del paciente geriátrico.

6) Uso de la silla de ruedas y los cuidados de la persona dependiente.

7) Cuidados paliativos.

8) Dignificación de la persona mayor.

9) Aspectos legales y bioéticos en el trato de la persona mayor.

Esta propuesta de diplomado ya fue entregada a la directora académica de la Facultad de Enfermería, quien, a su vez, lo ha turnado al área de Rectoría. En la ciudad de San Francisco de Campeche se percibe poco interés por parte de las instancias de educación y de Gobierno para realizar una capacitación dirigida a los cuidadores informales, por lo que es necesario que las instancias educativas asuman su compromiso social y promuevan este tipo de capacitación, la cual permita a las familias tener elementos necesarios para atender mejor a sus familiares con envejecimiento patológico y se promueva el autocuidado, pero sobre todo la importancia de gerontologizar a la sociedad. 


\section{Discusión}

A la luz de los resultados de este estudio, se comprueba lo expresado en la Segunda Asamblea Mundial sobre el Envejecimiento:

Capacitar y ofrecer incentivos a los profesionales de los servicios sociales y de la salud para que asesoren y guíen a las personas que están llegando a la vejez sobre los modos de vida saludables y el cuidado de la propia salud (ONU, 2003, p. 27)

Es necesario contar con profesionales capacitados. Aquí, las 18 personas que asistieron al taller mostraron su compromiso por aplicar lo aprendido con sus pacientes. El total, asimismo, expresó que muchos de los temas eran novedosos, de ahí el interés por continuar con esta formación encaminada a la atención de las personas mayores.

Este estudio tuvo como limitantes la falta de interés de los responsables de los establecimientos de asistencia social, al no permitir que asistiera su personal. Los que se ubican al interior del estado de Campeche, México, argumentaron que carecían de recursos económicos que les permitieran trasladarse a la ciudad de San Francisco de Campeche para tomar el taller. Otra limitante fue que los directivos de los establecimientos de asistencia social no hicieron extensiva la invitación a su personal, tal vez para evitar comprometerse con el pago de sus viáticos; la excepción fue el asilo de ancianos del municipio de Hopelchén, del cual asistieron dos personas, que cubrieron sus gastos de traslado, hospedaje y alimentación por todos los días del curso.

Por otro lado, este estudio tiene como fortaleza ser el primero que se realiza en la Facultad de Enfermería de la Universidad Autónoma de Campeche, México. Se trató de una unión de esfuerzos para aportar a la sociedad en el tema de la gerontologización de los profesionales de la salud que se desempeñan en el cuidado de pacientes en las etapas de la vejez y ancianidad. Sin embargo, la principal fortaleza es que los profesionistas que acudieron al taller ahora están mejor preparados para ejercer sus actividades en el trabajo y atención con calidad hacia personas en las últimas etapas de su vida.

Entre las debilidades presentadas en este estudio se ubica la de continuar con la línea de formación para gerontologizar a la sociedad y fortalecer sus conocimientos, no solo a los profesionistas del área de la salud, sino también incluir a los familiares, es decir, que el trabajo se realice en dos vertientes: dirigido a los cuidadores formales e informales, esto contribuirá a lograr la eficiencia de la atención de las personas. Otra área de oportunidad para 
futuras investigaciones es incluir el tema de la salud mental en la formación gerontogeriátrica.

\section{Conclusiones}

Como resultado del taller, se capacitó a 18 personas que cuidan y tienen contacto con personas mayores. Se analizó el perfil de los participantes, a partir de lo cual se obtuvo que, de los integrantes de la muestra, $28 \%$ son enfermeros, $28 \%$ gerontólogos, $12 \%$ gerocultores, $17 \%$ auxiliares de enfermería, $5 \%$ médicos, $5 \%$ licenciados en Economía y $5 \%$ cuenta con bachillerato. Sobre todo, se encontró que $71 \%$ tiene algún tipo de capacitación en el área de la salud para su función. Se diseñó y realizó un taller y una propuesta de diplomado para cuidadores informales.

La hipótesis se acepta, ya que $29 \%$ de la muestra no cuenta con cursos en el área de la atención del paciente geriátrico y 90 \% de los participantes desconocía las plataformas de capacitación en temas gerontogeriátricos. Por otro lado, la totalidad de los participantes resultó satisfecha con el taller. Por lo que es importante continuar en la formación de cuidadores de pacientes geriátricos y en el cuidado de su salud mental.

\section{Agradecimientos}

Se agradece a la directora académica de la Facultad de Enfermería de la Universidad Autónoma de Campeche por todas las facilidades otorgadas para el buen desarrollo de este estudio. De igual forma, se agradece la confianza en esta actividad depositada por todas las residencias geriátricas y a las autoridades de la Delegación Campeche del ISSSTE que permitieron la participación de su personal en el taller para cuidadores. 


\section{Referencias}

Cámara de Diputados del H. Congreso de la Unión. (2018). Ley de los Derechos de las Personas Adultas Mayores. México: Cámara de Diputados del H. Congreso de la Unión. Recuperado de http://www.diputados.gob.mx/LeyesBiblio/pdf/245_120718.pdf.

Consejo Estatal de Población (2018) Situación de las Personas Adultas Mayores en el Estado de Campeche. Gobierno del Estado de Campeche 2015 - 2021. Recuperado de http://www.coespo.campeche.gob.mx/phocadownload/AdultosMayores2018.pdf

Consejo Estatal de Población (2016) Proyecciones de la población de las entidades federativas y la República Mexicana. Proyecciones de los indicadores sociodemográficos del Estado de Campeche 2016 - 2050. Poder Ejecutivo del Estado de Campeche. Recuperado de http://www.coespo.campeche.gob.mx/index.php/13coespo/199-informacion-estadistica

Diario Oficial de la Federación [DOF]. (5 de octubre de 2010). NOM-004-SSA3-2012, Del expediente clínico. Diario Oficial de la Federación. Recuperado de http://dof.gob.mx/nota_detalle_popup.php?codigo=5272787.

Diario Oficial de la Federación [DOF]. (13 de septiembre de 2012). NOM-031-SSA3-2012, Asistencia social. Prestación de servicios de asistencia social a adultos y adultos mayores en situación de riesgo y vulnerabilidad. Diario Oficial de la Federación. Recuperado http://dof.gob.mx/nota_detalle.php?codigo=5267965\&fecha=13/09/2012.

Diario Oficial de la Federación [DOF]. (12 de septiembre de 2013). Norma Oficial Mexicana NOM-030-SSA3-2013, Que establece las características arquitectónicas para facilitar el acceso, tránsito, uso y permanencia de las personas con discapacidad en establecimientos para la atención médica ambulatoria y hospitalaria del Sistema Nacional de Salud. Diario Oficial de la Federación. Recuperado de https://www.dof.gob.mx/nota_detalle.php?codigo=5313974\&fecha=12/09/2013.

Instituto Nacional de Estadística y Geografía [INEGI]. (2015). Encuesta intercensal 2015. Comunicación social boletín de prensa, Núm. 524/15, 8 de diciembre de 2015 Aguascalientes, Ags., México. Página 2/4. Recuperado de https://www.inegi.org.mx/contenidos/programas/intercensal/2015/doc/especiales201 5_12_3.pdf 
Instituto Nacional de Estadística y Geografía [INEGI]. (2010). Censo de Población y Vivienda 2010. México: Instituto Nacional de Estadística y Geografía. Recuperado de https://www.inegi.org.mx/programas/ccpv/2010/.

Instituto Nacional de las Personas Adultas Mayores [INAPAM]. (2012). Modelos de atención gerontológica. México: Instituto Nacional de las Personas Adultas Mayores. Recuperado de http://www.inapam.gob.mx/work/models/Inapam/Resource/Documentos_Inicio/Lib ro_Modelos_de_Atencion_Gerontologica_(web).pdf.

MedlinePlus. (5 de diciembre de 2018). Cómo elegir un hogar para ancianos y convalecientes. MedlinePlus. Información de salud para usted. Recuperado de https://medlineplus.gov/spanish/ency/patientinstructions/000956.htm.

Millán, J. C. (2006). Principios de geriatría y gerontología. España: McGraw-Hill.

Morris, C. y Maisto, A. (2011). Introducción a la psicología (13. a ed.). México: Pearson Educación.

Organización de las Naciones Unidas [ONU]. (2003) Declaración Política y Plan de Acción Internacional de Madrid sobre el Envejecimiento. Nueva York, Estados Unidos: Naciones Unidas. Recuperado de https://social.un.org/ageing-workinggroup/documents/mipaa-sp.pdf. 
Revista Iberoamericana de las Ciencias de la Salud

\begin{tabular}{|l|l|}
\hline Rol de Contribución & Autor (es) \\
\hline Conceptualización & Jaqueline Guadalupe Guerrero Ceh \\
\hline Metodología & Jaqueline Guadalupe Guerrero Ceh \\
\hline Software & Maríajosé Sanchez De la Rosa \\
\hline Validación & Maríajosé Sanchez De la Rosa \\
\hline Análisis Formal & Jaqueline Guadalupe Guerrero Ceh \\
\hline Investigación & Jaqueline Guadalupe Guerrero Ceh \\
\hline Recursos & Jaqueline Guadalupe Guerrero Ceh \\
\hline Curación de datos & Maríajosé Sanchez De la Rosa \\
\hline $\begin{array}{l}\text { Escritura - Preparación del } \\
\text { borrador original }\end{array}$ & Jaqueline Guadalupe Guerrero Ceh \\
\hline $\begin{array}{l}\text { Escritura - Revisión y } \\
\text { edición }\end{array}$ & Jaqueline Guadalupe Guerrero Ceh \\
\hline Visualización & Jaqueline Guadalupe Guerrero Ceh \\
\hline Supervisión & Jaqueline Guadalupe Guerrero Ceh \\
\hline Proyectos & Jaqueline Guadalupe Guerrero Ceh \\
\hline Adquisición de fondos & Jaqueline Guadalupe Guerrero Ceh \\
\hline
\end{tabular}

